\title{
Compositional Equivalence of Grain from Multi-trait Drought- Tolerant Maize Hybrids to a Conventional Comparator: Univariate and Multivariate Assessments
}

\author{
Yun $\mathrm{Xu}^{\dagger}$ Royston Goodacre, ${ }^{* \dagger}$ and George G. Harrigan ${ }^{*} \S$ \\ ${ }^{\dagger}$ School of Chemistry, Manchester Institute of Biotechnology, University of Manchester, 131 Princess Street, Manchester M1 7ND, \\ United Kingdom \\ ${ }^{\S}$ Compositional Biology Center, Monsanto Company, 800 North Lindbergh Boulevard, St. Louis, Missouri 63167, United States
}

Supporting Information

\begin{abstract}
MON 87460 (D1) maize contains a gene that expresses the cold shock protein B (CSPB) from Bacillus subtilis to confer a yield advantage when yield is limited by water availability. This study evaluated the composition of grain from the D1-containing combined-trait maize hybrids D1 × NK603, D1 $\times$ MON $89034 \times$ NK603, and D $1 \times$ MON $89034 \times$ MON 88017. These stacks offer a combination of insect protection and herbicide tolerance traits. These hybrids were grown under well-watered and water-limited conditions at three replicated field sites across Chile during the 2006-2007 growing season. Compositional analyses included measurement of proximates, fibers, total amino acids, fatty acids, minerals, vitamins, raffinose, phytic acid, p-coumaric acid, and ferulic acid. The statistical analyses included an evaluation of the applicability of multiblock principal component analysis (MB-PCA) and ANOVA-simultaneous component analysis (ASCA) to studies when more than one experimental factor will contribute to compositional variability. Results from these multivariate procedures highlighted that water treatment was the greatest contributor to compositional variability and, as expected, confirmed that the grain of combinedtrait drought-tolerant hybrids was compositionally equivalent to that of conventional comparators as established by traditional statistical significance testing.
\end{abstract}

KEYWORDS: drought tolerance, GM corn, composition, multivariate analyses

\section{INTRODUCTION}

The drought-tolerant maize MON 87460 (D1) provides a yield benefit when yield is limited by water availability. ${ }^{1}$ D1 was produced by stable insertion of the coding sequence of cold shock protein B (CSPB) from Bacillus subtilis. Conventional breeding techniques have now developed the combined-trait products D $1 \times \mathrm{NK} 603$, D1 $\times$ MON $89034 \times$ NK603, and D $1 \times$ MON $89034 \times$ MON 88017. Each biotechnology-derived trait contributes specific benefits of drought tolerance (D1), herbicide tolerance (NK603), or insect resistance (MON 89034 and MON 88017) to the final product.

The compositional equivalence of crops improved through biotechnology-derived traits to their conventional counterparts has been consistently demonstrated. ${ }^{2-10}$ This observation extends to the single-event products D1, NK603, MON 89034, and MON 88017 that comprise the combined-trait products evaluated in this study. ${ }^{2-5}$ This equivalence substantiates the safety of these products and highlights the fact that transgene insertion has no meaningful impact on the nutritional quality of genetically modified (GM) products. The purpose of this study was to compare the composition of grain harvested from the combined-trait products to that of a near-isogenic conventional comparator when grown under well-watered and water-limited conditions as well as to provide further information on the effect of drought on composition.

In addition to confirming the equivalence of GM and non-GM crops, compositional studies have consistently demonstrated that other factors such as germplasm, environment, and management practices contribute more to compositional variability in levels of key nutrients and antinutrients than transgenic breeding. ${ }^{2-8}$ Graphical and statistical approaches that can provide meaningful evaluations of the relative impact of different factors to compositional variability may offer advantages over traditional frequentist testing. Bayesian as well as exploratory data analysis (EDA) approaches that may provide more meaningful explanations of compositional data than routinely misinterpreted significance testing have also been recently promoted. ${ }^{9-12}$

Multivariate approaches have also been considered. For example, principal variance component analysis ${ }^{13,14}$ has been used to provide summary graphics of the impact of multiple experimental factors on compositional variability in a study on GM cotton grown at multiple locations in the United States.

Here we provide an evaluation of the applicability of multiblock principal component analysis (MB-PCA $)^{15}$ and ANOVAsimultaneous component analysis (ASCA). ${ }^{16}$ The goal was to assess methods that can handle interacting factors and so present the impact of location, water treatment, and transgenic breeding on compositional variability more accurately. In addition, we wish to assess whether such approaches could complement, or provide advantages over, more traditional univariate approaches.

Received: April 28, 2014

Revised: August 22, 2014

Accepted: August 31, 2014

Published: September 1, 2014 
Grain was harvested from three replicated field sites across Chile during the 2006-2007 season. Twelve different conventional maize hybrids were included to provide supplementary data on compositional variability due to field location and water treatment. All field sites utilized a strip-plot design with three replicates per site, with water treatment (well-watered or waterlimited) as the whole plot factor and substance type as the subplot factor. Compositional analyses included measurement of proximates, fibers, total amino acids, fatty acids, minerals, vitamins, raffinose, phytic acid, $p$-coumaric acid, and ferulic acid.

\section{MATERIALS AND METHODS}

Maize Samples for Compositional Analysis. The droughttolerant maize MON 87460 (D1) provides a yield benefit when yield is limited by water availability. ${ }^{1} \mathrm{D} 1$ was produced by stable insertion of the coding sequence of cold shock protein B (CSPB) from B. subtilis. Conventional breeding techniques have now developed the combinedtrait products D1 × NK603, D1 × MON $89034 \times$ NK603, and D1 $\times$ MON $89034 \times$ MON 88017. Each biotechnology-derived trait contributes specific benefits of drought tolerance (D1), herbicide tolerance (NK603), or insect resistance (MON 89034 and MON 88017) to the final product.

Seed of the drought-tolerant combined-trait products and a nearisogenic conventional control were planted in the winter of 2006 at three sites in major maize-producing regions of Chile. Seeds from four different commercial conventional hybrids were also included at each field site (for a total of 12 unique hybrids). Locations of the field sites were as follows: Colina, Region Metropolitana; Calera de Tango, Region Metropolitana; and Lumbreras, Region Metropolitana. All field sites utilized a strip-plot design with three replicates per site, with water treatment (well-watered or water-limited) as the whole plot and substance type as the subplot. The whole-plot factor was arranged as a randomized complete block design. The strip-plot factor consisted of the different maize varieties. The well-watered treatment was managed to provide optimal grain yield. The water-limited treatment was managed to impose a drought stress by withholding water during the late vegetative through early grain fill growth stages (i.e., approximately V10 through R2). The use of a split-plot design allowed comparisons of the drought-tolerant hybrids to the control under two separate irrigation regimens. Tissue was collected from all three blocks for each treatment; tissue from the different commercial references grown at each site was collected from a single block for each treatment. This resulted in a total of 90 test and control samples ( 5 substances $\times 3$ biological replicates $\times 3$ field sites $\times 2$ water treatments) and 24 commercial reference samples ( 4 substances $\times 1$ biological replicate $\times 3$ field sites $\times 2$ water treatments).

The genetic purity of the maize plants was maintained by bagging the tassels and ear shoots at anthesis and self-pollinating each plant by hand. Forage was collected at the late dough/early dent stage, and the grain was collected at normal kernel maturity (Supporting Information). After harvest, samples were shipped to Monsanto, St. Louis, MO, USA. The samples were ground to a fine powder in the presence of dry ice and maintained frozen at $-20{ }^{\circ} \mathrm{C}$ until required for compositional analysis. The identity of forage and grain samples was based on sample handling records and Southern blot or polymerase chain reaction analyses of genomic DNA isolated from grain tissue.

Compositional Analyses. Components assessed in grain samples included proximates (protein, fat, ash, and moisture), carbohydrates by calculation, acid detergent fiber (ADF), neutral detergent fiber (NDF), total dietary fiber (TDF), total amino acid composition, fatty acid composition, minerals (calcium, copper, iron, magnesium, manganese, phosphorus, potassium, sodium, zinc), vitamins [vitamin B1 (thiamin), vitamin B2 (riboflavin), vitamin B6 (pyridoxine), vitamin E, niacin, folic acid], furfural, raffinose, phytic acid, $p$-coumaric acid, and ferulic acid. Components assessed in forage samples (Supporting Information) included proximate, carbohydrates by calculation, ADF, NDF, calcium, and phosphorus.

Compositional analyses were performed at Covance Laboratories, Inc. (Madison, WI, USA). Brief descriptions of the methods utilized for the analyses are described in refs $2-5$. To control and minimize bias, the samples produced from the three field sites were analyzed in a randomized order.

Statistical Analysis of Composition Data. In all, 68 different grain components were measured. The following 16 analytes with $>50 \%$ of the observations at or below the limits of quantitation (LOQ) of the assay were excluded from statistical analysis: sodium, furfural, caprylic acid, capric acid, lauric acid, myristic acid, myristoleic acid, pentadecanoic acid, pentadecenoic acid, palmitoleic acid, heptadecanoic acid, heptadecenoic acid, $\gamma$-linolenic acid, eicosadienoic acid, eicosatrienoic acid, and arachidonic acid. The LOQs for sodium, furfural, and fatty acids were $0.5 \mathrm{ppm}, 100 \mathrm{ppm}$, and $0.004 \%$, respectively. Except for moisture and fatty acids, all component values were converted from a fresh weight to a dry weight basis and into their respective units as presented in Tables 1-6 and Supplementary Table 1 (Supporting Information).

PRESS residuals were used to identify outliers. Extreme data points that were outside the \pm 6 studentized PRESS residual range were considered for exclusion, as outliers, from the final analyses. This resulted in a single copper value recorded for one of the commercial conventional reference hybrids grown at the Colina site being excluded.

Compositional components were statistically analyzed using a mixedmodel analysis of variance from each of the three sites and from the combination of all sites. The combined-site analysis used the model

$$
\begin{aligned}
Y_{i j k l}= & U+L_{i}+B(L)_{i j}+T_{k}+L T_{i k}+T B(L)_{i j k}+S_{l}+T S_{k l} \\
& +L S_{i l}+L T S_{i k l}+\mathrm{e}_{i j k l}
\end{aligned}
$$

where $Y_{i j k l}=$ unique individual observation, $U$ = overall mean, $L_{i}=$ random location effect, $B(L)_{i j}=$ random block within location effect, $T_{k}=$ irrigation treatment effect, $L T_{i k}=$ random location by treatment interaction effect, $T B(L)_{i j k}=$ random treatment by block within location interaction effect, $S_{l}=$ substance effect, $T S_{k l}=$ treatment by substance interaction effect, $L S_{i l}=$ random location by substance interaction effect, $L T S_{i k l}=$ random location by treatment by substance interaction effect, and $\mathrm{e}_{i j k l}=$ residual error.

For each component analysis, mean comparison tests of each of the drought-tolerant hybrids to the conventional control within each water treatment were conducted.

SAS software was used to generate all summary statistics and perform all analyses (SAS Institute, 2002-2003). All statistical analyses were conducted by Certus International, Inc. (Chesterfield, MO, USA).

\section{MULTIVARIATE ANALYSIS}

To assess the effect of each factor better, MB-PCA and ASCA were applied to the data. Both models are extensions of the classical PCA model and can separate variances caused by multiple factors and thus provide a better view of the effect of each factor of interest.

For MB-PCA, the original compositional data were repartitioned into a series of blocks according to the experiment design (i.e., blocking). Through such blocking, one particular factor would become baseline and no longer have an effect on the data, whereas the other factor(s) would become a common trend across all of the blocks and have their influence enhanced, assuming that the samples are properly matched and the effect of the factor(s) of interest is consistent across different blocks. This is clearly a useful approach to analyze a system with multiple and potentially interacting factors. A MB-PCA model comprises three main components, (1) super scores, (2) block scores, and (3) block loadings, where the common trend across all of the blocks is shown in the super scores, the individual patterns of the blocks are shown in the block scores, and the variable contributions to the pattern shown by the MB-PCA model can be found in the block loadings.

ASCA is another recently proposed model aimed at the analysis of data with multiple factors. In ASCA, the data matrix is decomposed into the sum of a series effect matrices and each effect matrix contains the level averages for each factor, which represents the effect of that particular factor. The variations that cannot be explained by the model are put into the residual matrix $\boldsymbol{\varepsilon}$. In this study there are three factors of interest, and thus the ASCA model is written as

$$
\boldsymbol{X}=1 \times \boldsymbol{m}^{T}+\boldsymbol{X}_{\mathrm{i}}+\boldsymbol{X}_{1}+\boldsymbol{X}_{\mathrm{s}}+\boldsymbol{X}_{(\mathrm{il})}+\boldsymbol{X}_{(\mathrm{is})}+\boldsymbol{X}_{(\mathrm{ls})}+\boldsymbol{X}_{(\mathrm{ils})}+\boldsymbol{\varepsilon}
$$






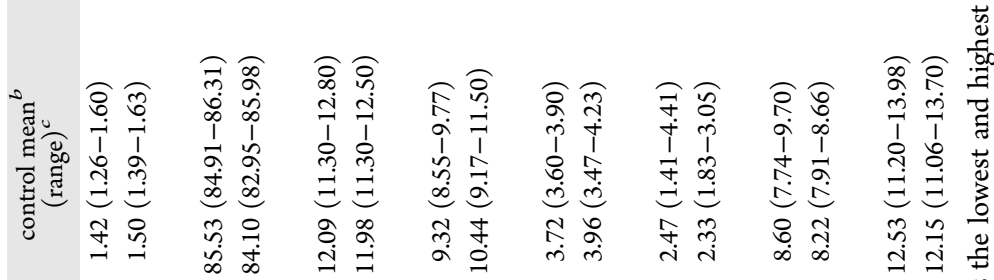

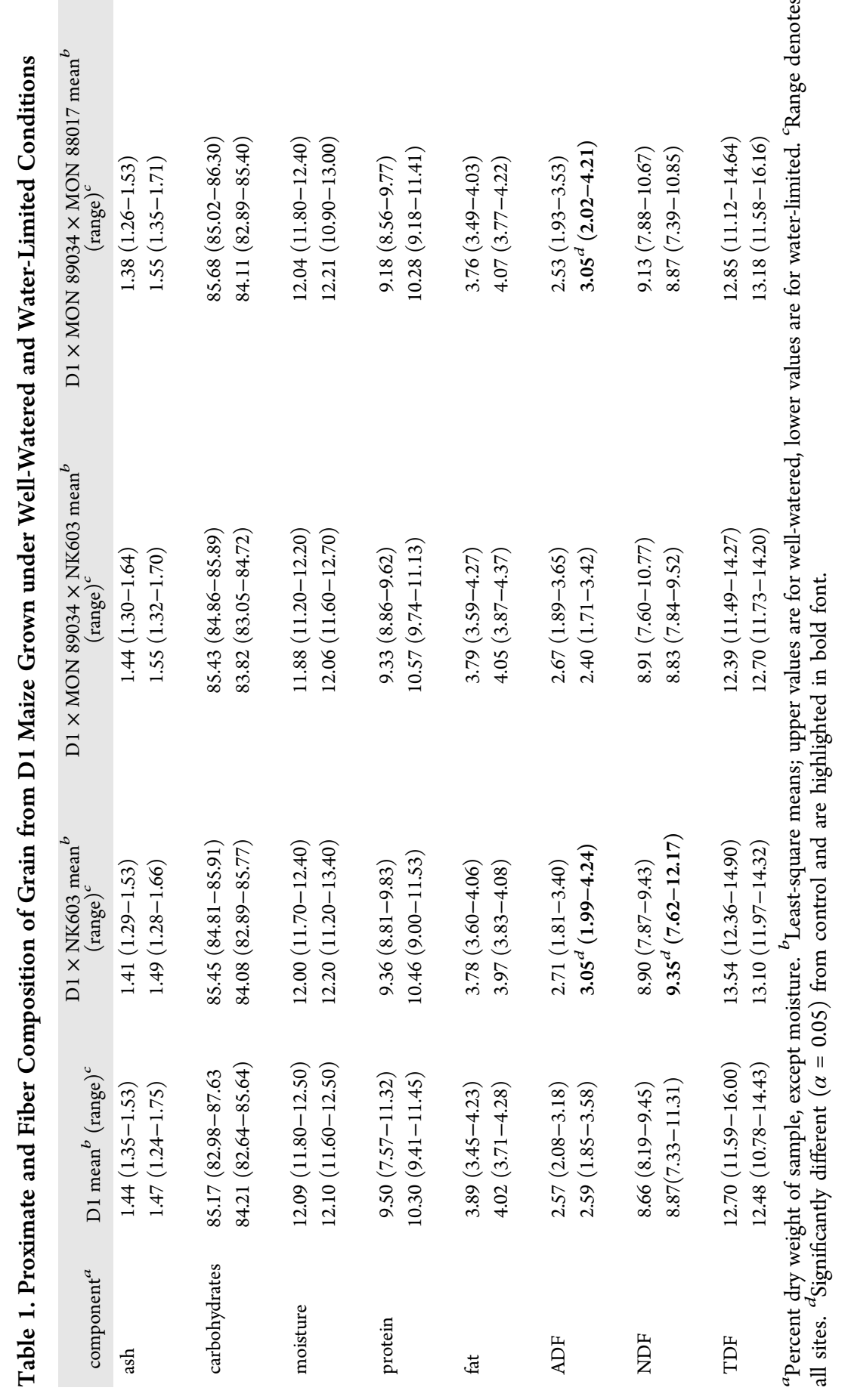


where $\boldsymbol{m}^{T}$ is the mean vector of the full matrix; 1 is a column vector of ones; $\boldsymbol{X}_{\mathrm{i}}, \boldsymbol{X}_{1}$ and $\boldsymbol{X}_{\mathrm{s}}$ are the effect matrices of the three factors, namely, irrigation conditions, locations, and substances, respectively. $\boldsymbol{X}_{(\mathrm{il})}, \boldsymbol{X}_{(\mathrm{is})}$, $\boldsymbol{X}_{(\mathrm{ls})}$, and $\boldsymbol{X}_{(\mathrm{ils})}$ are the interaction matrices between these factors, respectively. PCA was performed on each effect matrix, respectively, to calculate loadings, and the score of each effect matrix was obtained by adding the residual matrix $\boldsymbol{\varepsilon}$ back to the effect matrix and projected into the PC space via the corresponding loadings. For example, the score of the effect matrix of the irrigation condition $T_{\mathrm{i}}$ factor is given as

$$
\boldsymbol{X}_{\mathrm{i}}=\left(\boldsymbol{\Gamma}_{\mathrm{i}}\right) \times \boldsymbol{P}_{\mathrm{i}}^{T}
$$

where $\boldsymbol{\Gamma}_{\mathrm{i}}$ and $\boldsymbol{P}_{\mathrm{i}}$ are the scores and loadings matrix obtained by PCA performed on $\boldsymbol{X}_{i}$.

$$
\boldsymbol{T}_{\mathrm{i}}=\left(\boldsymbol{X}_{\mathrm{i}}+\boldsymbol{\varepsilon}\right) \times \boldsymbol{P}_{\mathrm{i}}
$$

Unlike MB-PCA, ASCA is essentially a supervised technique; thus, an appropriate validation procedure is needed. In this paper, we employed a permutation test-based validation procedure proposed by Zwanenburg et al. ${ }^{16}$ In the validation, the magnitude of the effect of the factor of interest is expressed as the sum of squares (SSQs) of $\boldsymbol{\Gamma}$. A total number of $n$ permutations (in this study $n=1000$ ) are performed, and in each permutation the labels of the samples are randomly permutated, ASCA is performed on the data using the permuted label, and the SSQs of $\Gamma$ are recorded. All of the SSQs of $\boldsymbol{\Gamma}$ form a null distribution, and the SSQ of $\boldsymbol{\Gamma}$ calculated from the ASCA model using the known labels (i.e., the observed SSQ) is then compared with the null distribution. An empirical $p$ value can then derived by counting the number of permutations that obtained equal or higher SSQs than the observed SSQ.

Compositional data were autoscaled so that each variable had zero mean and unit standard deviation before being subjected to MB-PCA and ASCA. All of the multivariate analyses were conducted using inhouse scripts written in MATLAB R2012a environment (Mathworks, Natick, MA, USA).

\section{RESULTS AND DISCUSSION}

Univariate Analysis. Tables 1-6 show means and ranges of grain components combined across all sites (the combinedsite analysis) for all maize hybrids and for both well-watered and water-limited treatments. Forage data are presented in Supplementary Table 1 (Supporting Information). Individual site data for the conventional control are presented in Supplementary Tables 2-8. Overall, the combined-site analysis of the grain data was characterized by extremely few significant differences $(\alpha=0.05)$ between the drought-tolerant combinedtrait products and the conventional control (and in all Tables the statistically relevant differences are highlighted in bold font). From a total of 416 comparisons (comparison of 4 test substances to the control at 2 different water treatments $\times 52$ analytes $)$ only $23(5.53 \%)$ were significant $(\alpha=0.05)$. These included only 9 comparisons from the well-watered treatments and 14 from the water-limited treatments. Clearly, given the paucity of observed differences, no meaningful trends were observed that would distinguish drought-tolerant hybrids from conventional control. This is described in more detail below.

Proximate and Fiber Composition. Levels of proximates and fiber were similar across all of the drought-tolerant hybrids and the conventional control under both water treatments (Table 1). No differences $(\alpha=0.05)$ between mean values of proximates and fiber of the drought-tolerant hybrids and the conventional control grown under well-watered conditions were observed. Within the water-limited treatment, differences $(\alpha=0.05)$ from the control $\mathrm{ADF}$ and NDF mean values were observed for D1 $\times$ NK603 and from control ADF mean values for D1 $\times$ MON $89034 \times$ MON 88017. For D $1 \times$ NK603, the magnitudes of the differences in mean values for $\mathrm{ADF}$ and $\mathrm{NDF}$ were 0.72 and $1.13 \% \mathrm{dwt}$, respectively. For D1 $\times$ MON $89034 \times$ MON 88017, the magnitude of the differences in mean values for ADF was $0.72 \%$ dwt. Given the known variability of grain fiber ${ }^{2-5,7}$ this was not considered a meaningful difference. Our results, however, did suggest that, for all maize hybrids, the levels of proximate and fibers differed consistently between the two irrigation treatments. Mean values, when combined across all samples, for ash, protein, and fat were $\sim 5, \sim 11$, and $\sim 6 \%$ higher, respectively, under water-limited conditions relative to well-watered conditions in the combined-site analyses. Conversely, mean values for $\mathrm{ADF}(\sim 6 \%), \mathrm{NDF}(\sim 5 \%)$, and TDF $(\sim 3 \%)$ were lower under water-limited conditions.

Total Amino Acid Composition. No differences $(\alpha=0.05)$ in mean values of amino acids of the drought-tolerant hybrids and the conventional control grown under well-watered conditions were observed (Table 2). Under water-limited conditions, differences were observed for methionine in D1 $\times$ NK603 and D $1 \times$ MON $89034 \times$ NK603 (see Table 2). The overall lack of differences is clearly related to the lack of differences in protein levels noted above.

Fatty Acid Composition. Under well-watered conditions, differences $(\alpha=0.05)$ between the drought-tolerant combinedtrait products were observed for palmitic acid, linoleic acid, and eicosenoic acid in D1 $\times$ NK603; for oleic acid and eicosenoic acid in D1 $\times$ MON $89034 \times$ NK603; and for palmitic acid in D $1 \times$ MON $89034 \times$ MON 88017 (Table 3). Under waterlimited conditions, significant differences were observed for eicosenoic acid in D1, palmitic acid in D1 $\times$ NK603, and palmitic acid and linolenic acid in D1 × MON $89034 \times$ MON 88017 . All differences were characterized by small mean differences, and no trends in differences between the different D1 hybrids and the conventional control were observed. There is little in the scientific literature on the effect of water treatment or drought on fatty acid composition. The data presented in Table 3 also provide some of the first scientific information that fatty acid composition is not greatly affected by water limitation.

Vitamin Composition. Overall, levels of vitamins were similar across all of the drought-tolerant hybrids and the conventional control under both water treatments (Table 4). Under well-watered conditions, significant differences $(\alpha=0.05)$ were observed for vitamin $\mathrm{E}$ in D1 $\times$ MON $89034 \times$ NK603 and for thiamin (vitamin B1) and pyridoxine (vitamin B6) in D1 $\times$ MON $89034 \times$ MON 88017. Under water-limited conditions, differences $(\alpha=0.05)$ were observed for niacin and riboflavin (vitamin $\mathrm{B} 2$ ) in $\mathrm{D} 1 \times \mathrm{NK} 603$; for vitamin $\mathrm{E}$ in $\mathrm{D} 1 \times \mathrm{MON}$ $89034 \times \mathrm{NK} 603$; and for thiamin and vitamin $\mathrm{E}$ in $\mathrm{D} 1 \times \mathrm{MON}$ $89034 \times$ MON 88017.

To our knowledge, there is little literature on the effect of abiotic stress on grain levels of maize vitamins. In the combinedsite analysis, mean values for most vitamins were higher under water-limited conditions relative to well-watered conditions. These included niacin, vitamin B2 (riboflavin), vitamin B1 (thiamin), and vitamin $\mathrm{E}$, which were increased $\sim 11, \sim 14, \sim 4$, and $\sim 10 \%$, respectively, upon water restriction. In contrast to the above results, combined-site analysis revealed that mean values for vitamin B6 (pyridoxine) were lower $(\sim 11 \%)$ under waterlimited conditions relative to well-watered conditions.

Antinutrient and Secondary Metabolite Composition. No differences $(\alpha=0.05)$ between the drought-tolerant combined-trait products and the control for phytic acid, raffinose, ferulic acid, and $p$-coumaric acid were observed (Table 5).

Mineral Composition. Overall, levels of minerals were similar across all of the drought-tolerant hybrids and the conventional control under both water treatments (Table 6). No differences 


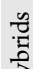

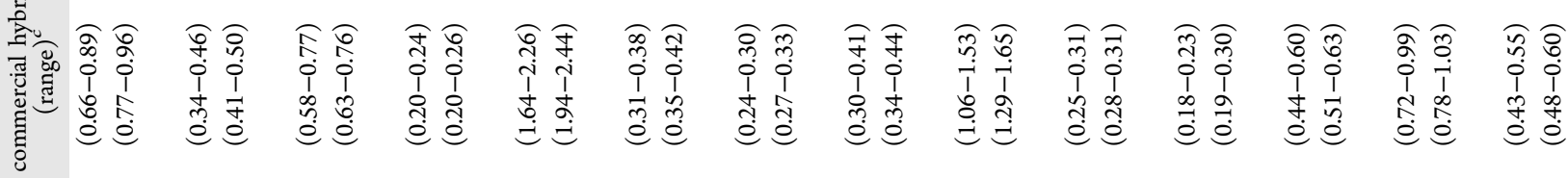

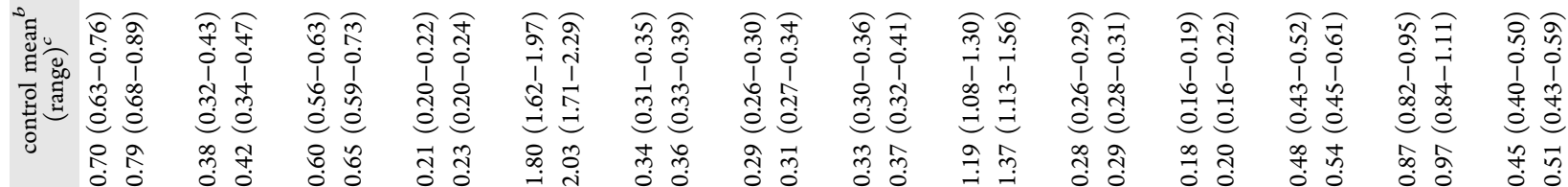
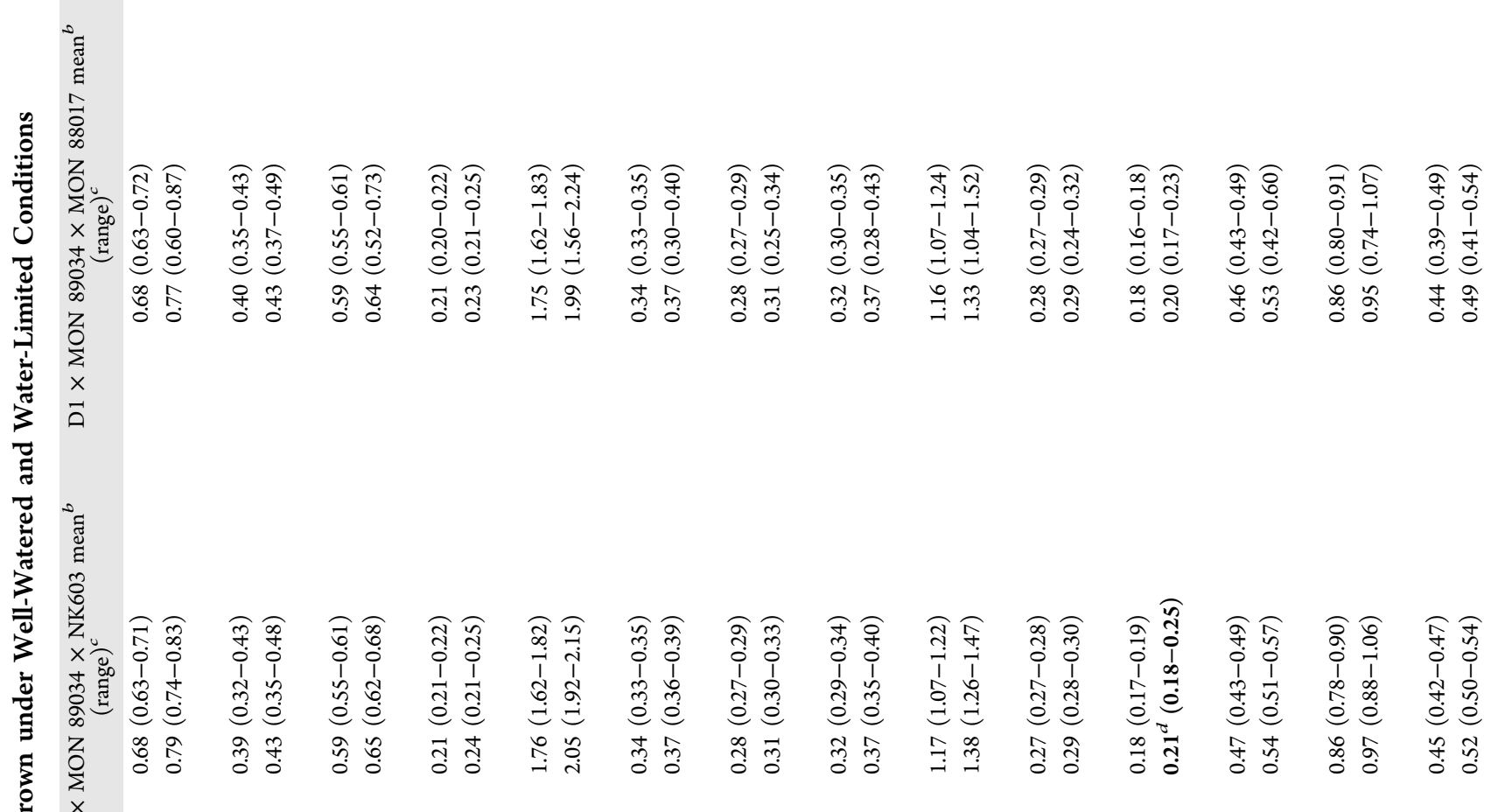

赵

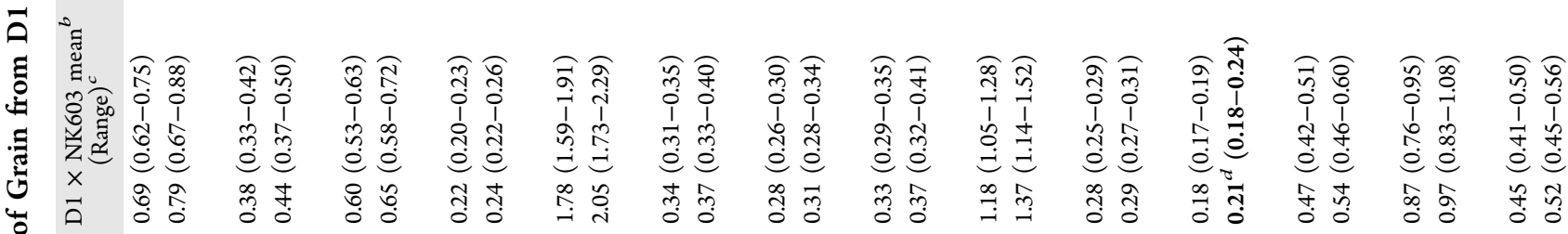

。ำ

: 


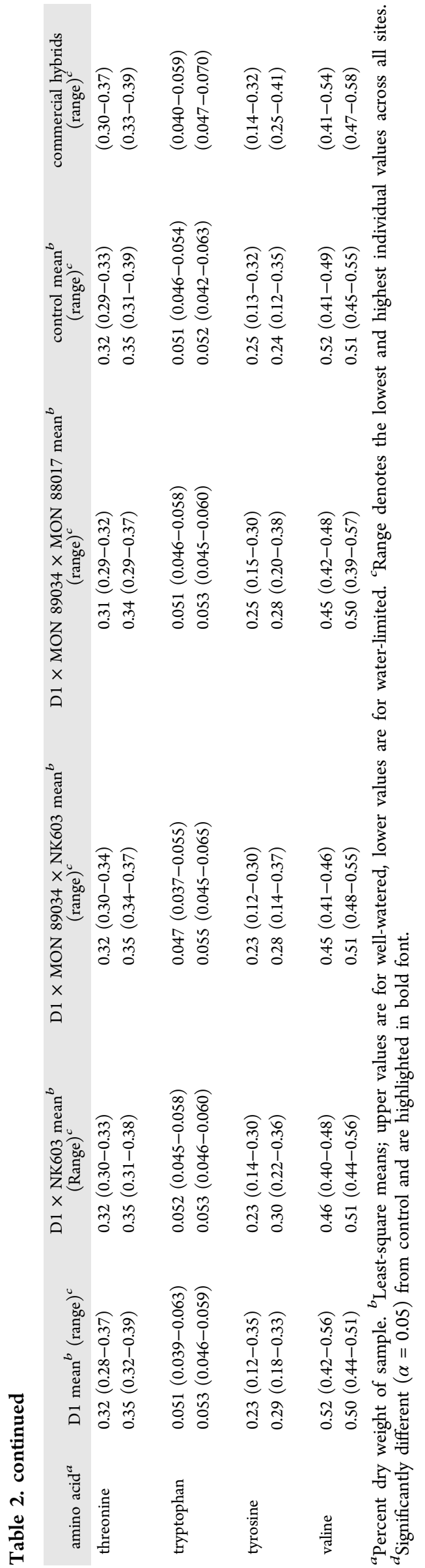

( $\alpha=0.05)$ in mean values of minerals of the drought-tolerant hybrids and the conventional control grown under well-watered conditions were observed (see Table 6). Within the water-limited treatment differences were observed for potassium in $\mathrm{D} 1 \times \mathrm{MON}$ $89034 \times$ NK603.

Mean values for all tested minerals, with the exception of $\mathrm{K}$, were higher $(\sim 8-15 \%)$ under water-limited conditions relative to well-watered conditions in the combined site analyses. There are few reports on the effect of limited water availability on mineral composition in maize grain. A three year study of tropical varieties subjected to preanthesis drought concluded that there was no significant impact of water restriction. ${ }^{17}$ Restricting water availability after silking also showed no effect on the levels of measured minerals ( $\mathrm{P}$ and $\mathrm{K}$ ) despite a $\sim 33 \%$ grain yield reduction. ${ }^{18}$ The changes observed imply that water deficit affects mineral composition in grain only modestly, an observation consistent with results from other peer-reviewed studies. ${ }^{17,18}$

Multivariate Analysis. The original focus of the study was a comparison of grain composition of the D1 maize hybrids to a near-isogenic conventional comparator. Review of the data presented in Tables 1-6 and in the Supporting Information clearly suggested, however, that water treatment and location contributed more to compositional variation than genetic modification. We were therefore interested in reviewing multivariate options to present meaningful visualization and interpretation of different sources of variation. PCA is one of the most popular tools for exploratory analysis, but it is not always effective when an experiment comprises multiple influential factors such as are present in our study. We initially performed PCA on the full data matrix, and the results appeared to be a complex mixture of the effects of all three factors (substance, location, and water treatment), and no obvious pattern could be observed (data not shown). We therefore attempted to use two different PCA variants, MB-PCA and ASCA, to better discern the effects of each factor.

MB-PCA. To better assess the effect of each factor, we created a series of MB-PCA models by repartitioning (i.e. blocking) the data in different ways so that one particular factor became a background and the effect of the factor of interest became a common trend across all blocks. Blocking was performed as follows.

(1) Three-Locations Blocking, with Each Block Containing Both Irrigation Conditions. The scores plots from this MB-PCA model are shown in Figure 1. These clearly show that water treatment conditions had a significant influence on the data. On the super scores (Figure 1a), a clear separation between the wellwatered condition (wet) and water-limited (dry) condition was seen across the first PC. The block scores (Figure $1 \mathrm{~b}-\mathrm{d}$ ) showed similar patterns for all three locations. In addition, the loadings (Figure 2) of the three blocks were highly similar to each other, suggesting that water treatment had a similar impact on grain composition, regardless of location.

Consistent with results from the univariate analysis, no clear separation between the different substances was observed in the scores plots of this model. Two further MB-PCA models were computed, which also blocked according to locations but contained only samples from the same water treatment (either wet or dry, respectively); results of these two models again showed no sign of separation between different substances (data not shown).

(2) Five-Substances Blocking Where the Data Were Repartitioned into Five Blocks, One for Each Substance. We first incorporated samples from both water treatments into each block; the super scores plot of the MB-PCA model is given in 


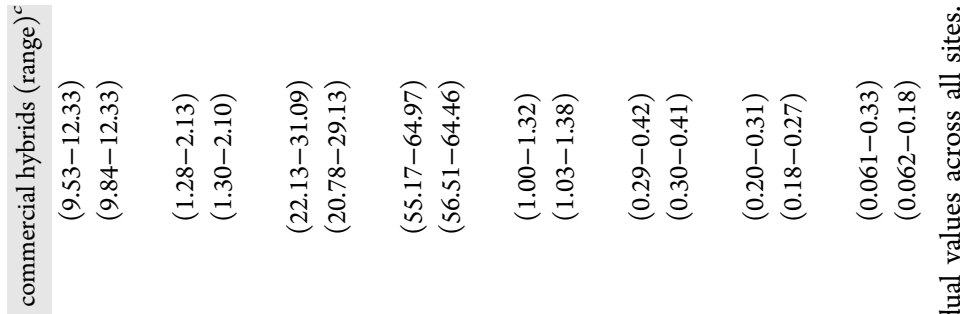

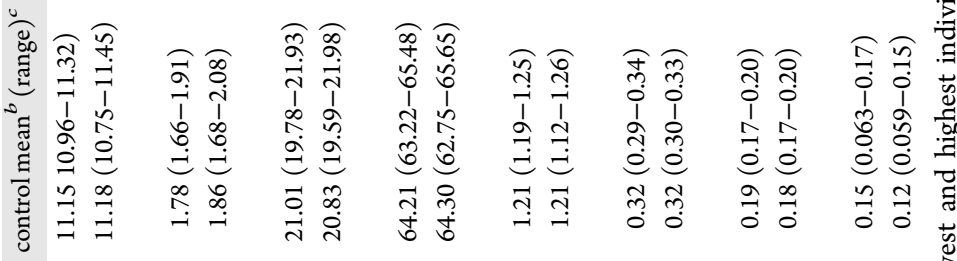

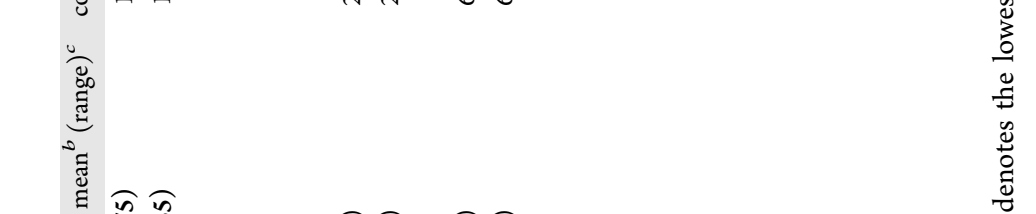

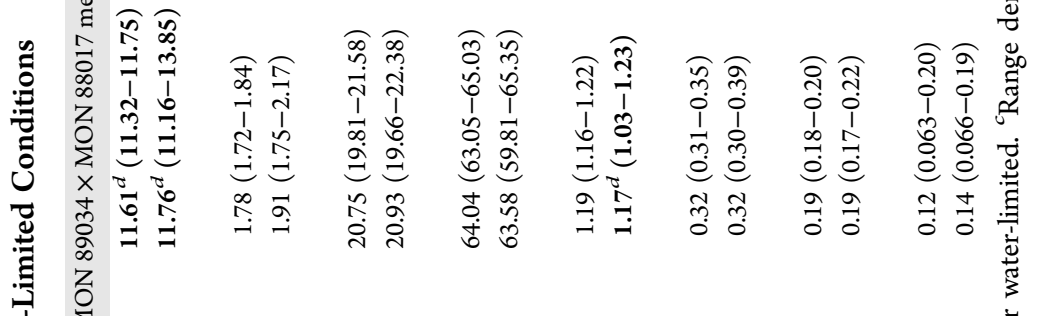

:

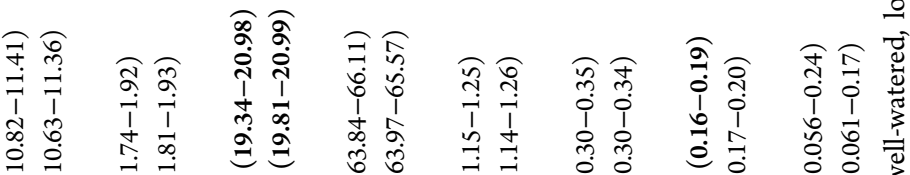

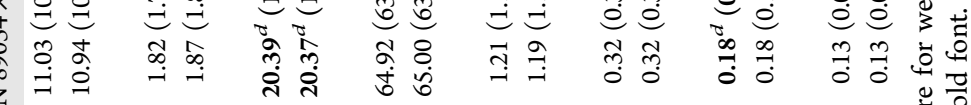

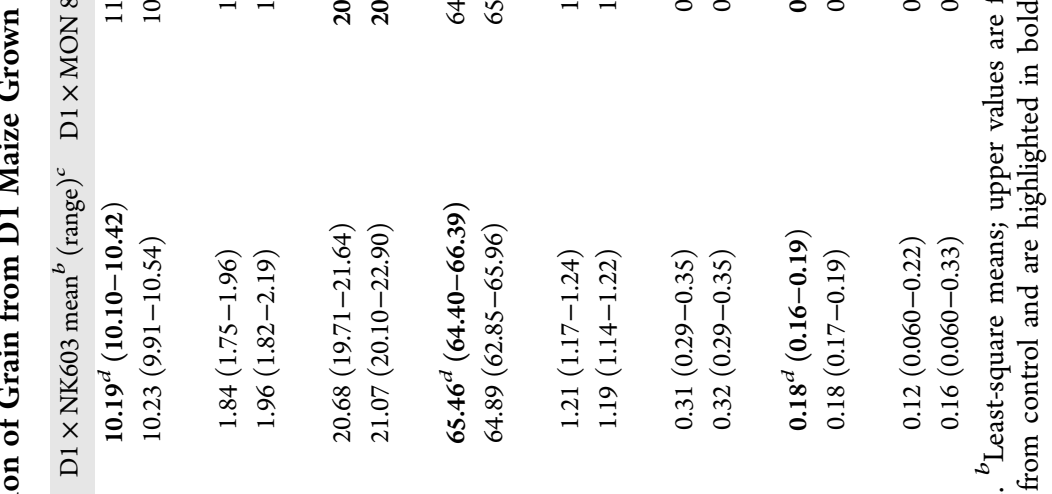

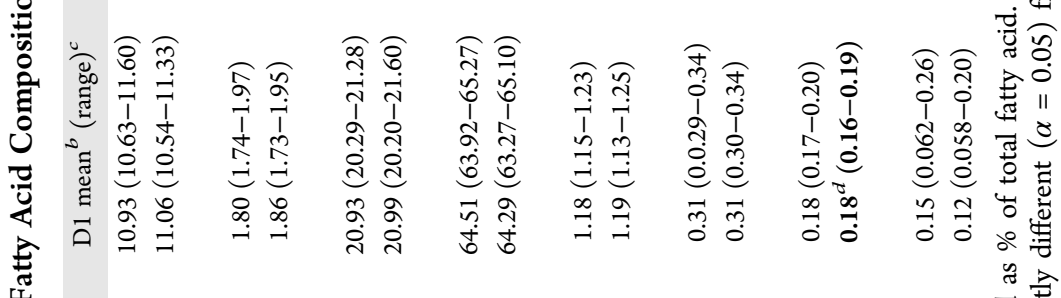






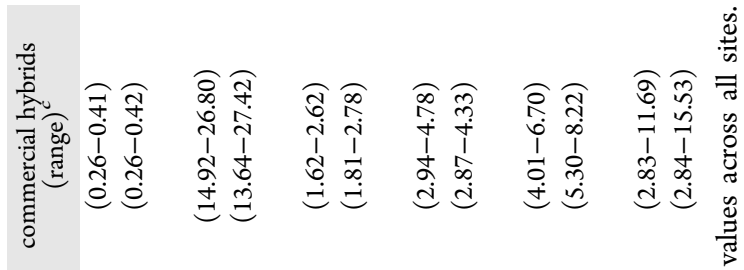

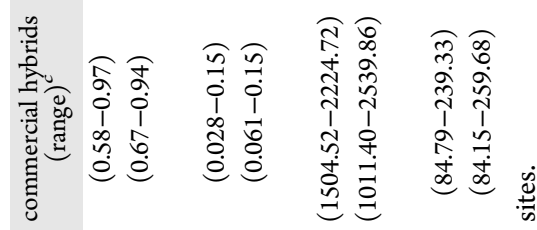

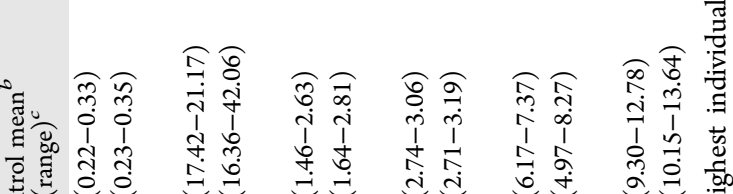

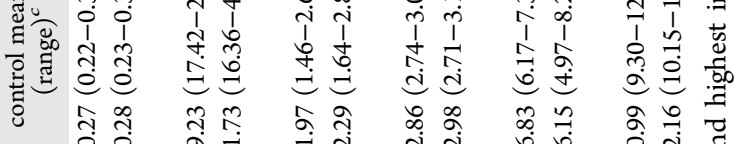

तิ

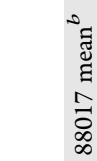

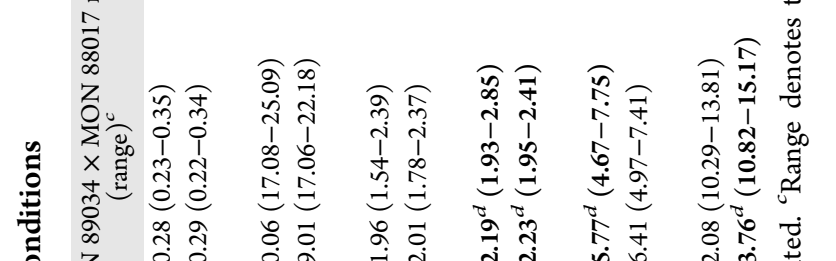

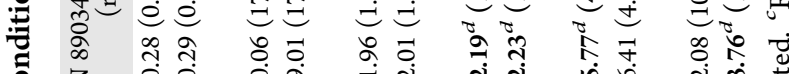

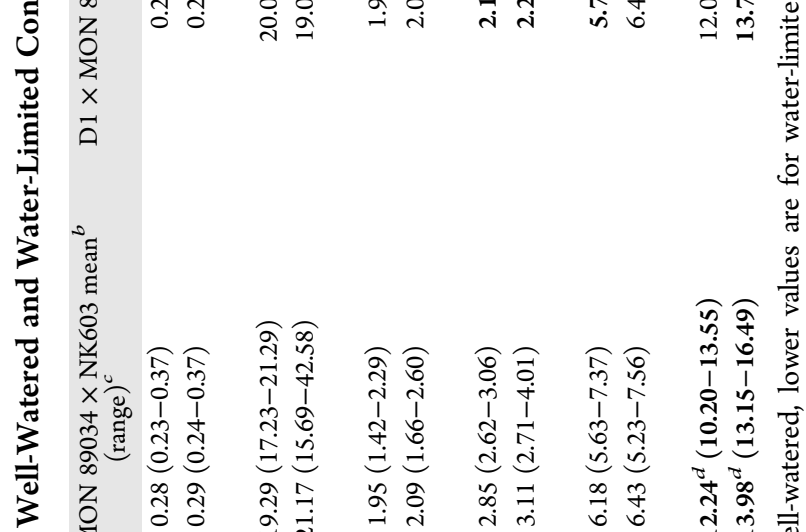

离

范

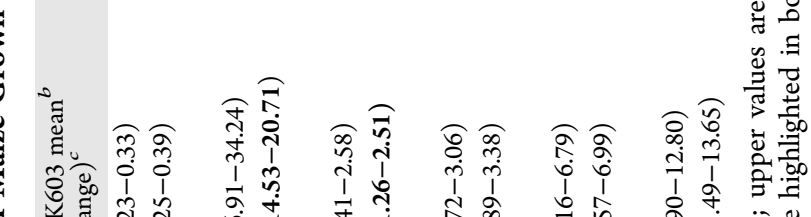

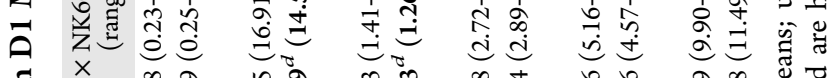

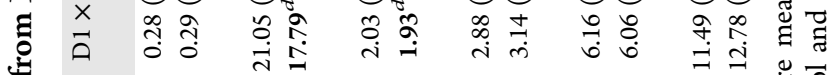

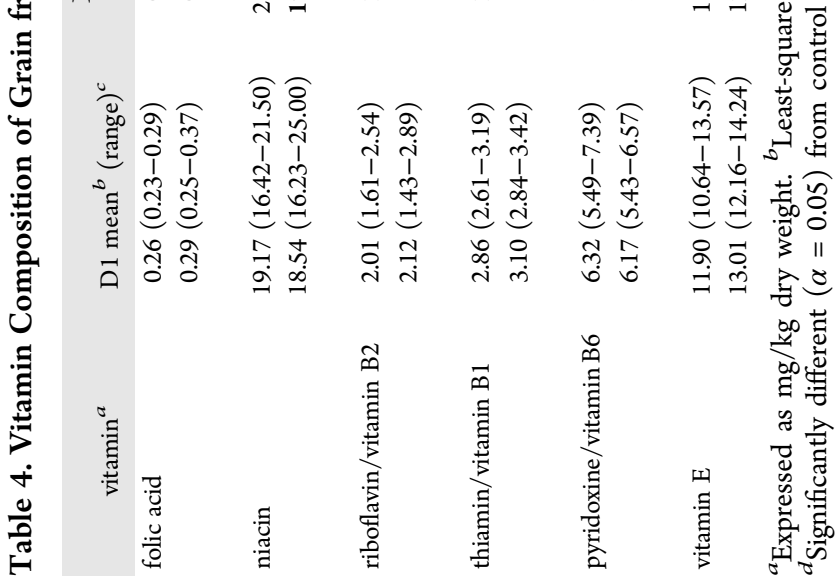



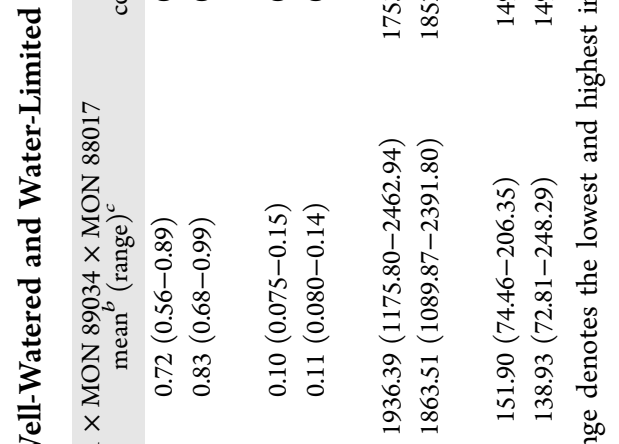

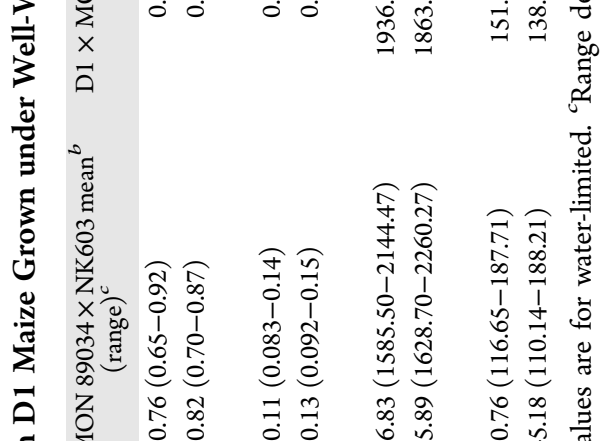

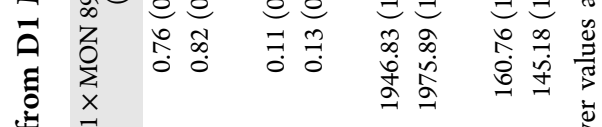

$\vec{a}$






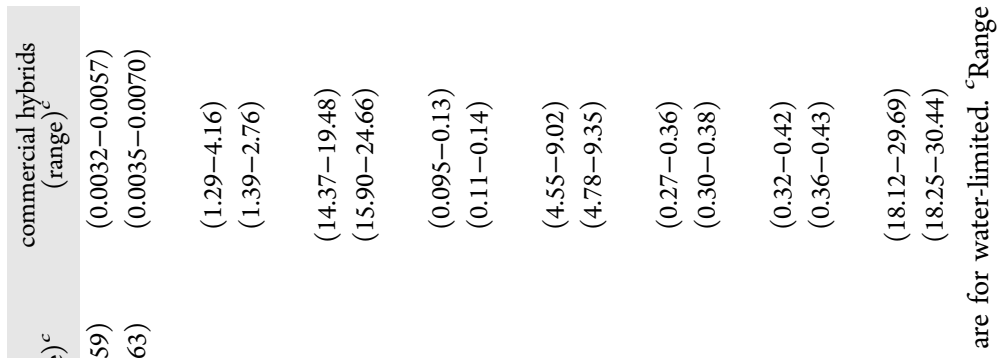



1

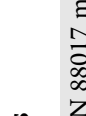

党

㟒



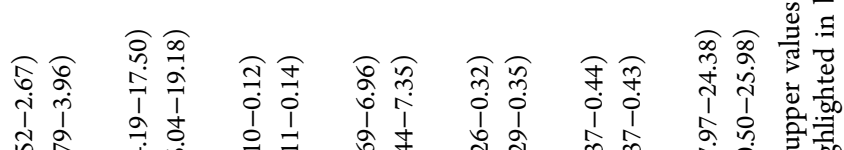

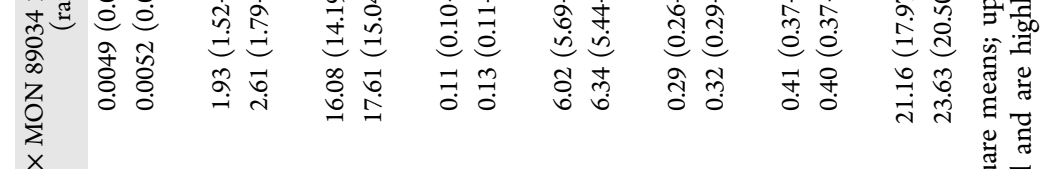

青

$\overrightarrow{0}$

苛

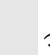

है

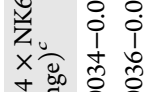

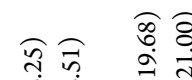

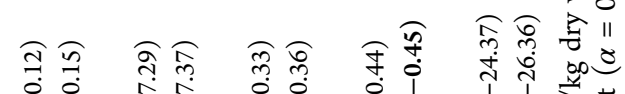

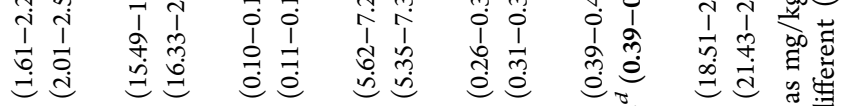

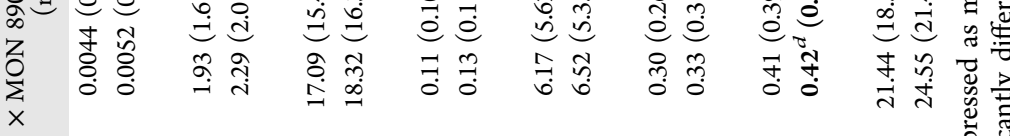

है

苞

$\vec{A}$

范

政



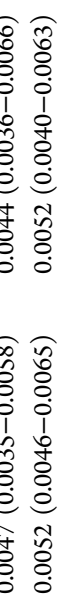

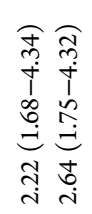

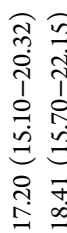

I

के

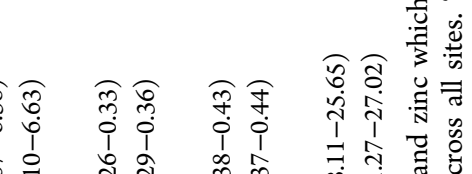

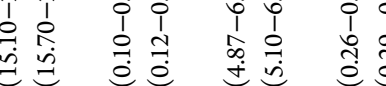

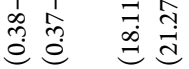

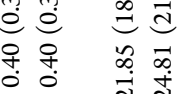


Blocking according to locations, both conditions, super scores


Figure 1. MB-PCA performed on the location blocking data set: (a) super scores plot; (b) block scores plot on the site CL block; (c) block scores plot on the site CT block; (d) block scores plot on the site LUM block. TEV, total explained variance.

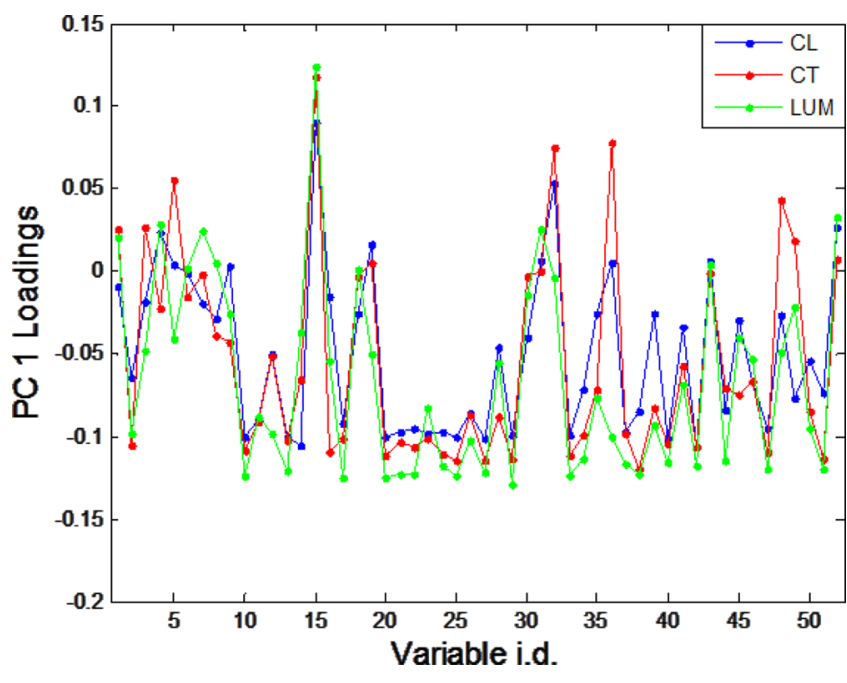

Figure 2. Loading plots from MB-PCA. The colors represent the different sites: (blue) site CL; (red), site CT; (green) site LUM. The list of variables (analytical components) is presented in Supporting Information.
Figure 3a. PC 1 clearly separated the two water treatments, and PC 2 separated (albeit with some overlap) the three sites along its axis. This suggested that water treatment had a stronger influence on composition compared to location differences. The super scores plot also showed that separation between the three different locations was better under well-watered conditions than under water-limited conditions. To confirm this, we built another two MB-PCA models using substances blocking, where each block contained only one water treatment, that is, wet or dry only. The super scores of the MB-PCA model based on well-watered samples (Figure $3 \mathrm{~b}$ ) indeed showed a much better separation than the MB-PCA model based on water-limited samples (Figure 3c).

ASCA. The MB-PCA models confirmed a lack of substance effect consistent with results from the univariate analysis. On the basis of different blocking strategies, the MB-PCA models further suggested that water treatment had the greatest impact on grain composition. Locations also had an effect, albeit weaker than that of water treatment. These location effects appeared to be greater under well-watered conditions than under water-limited conditions. We tested this observation by employing an ASCA model. The scores plots of the three submatrices of the ASCA model are given in Figure 4. It can be seen that separation 

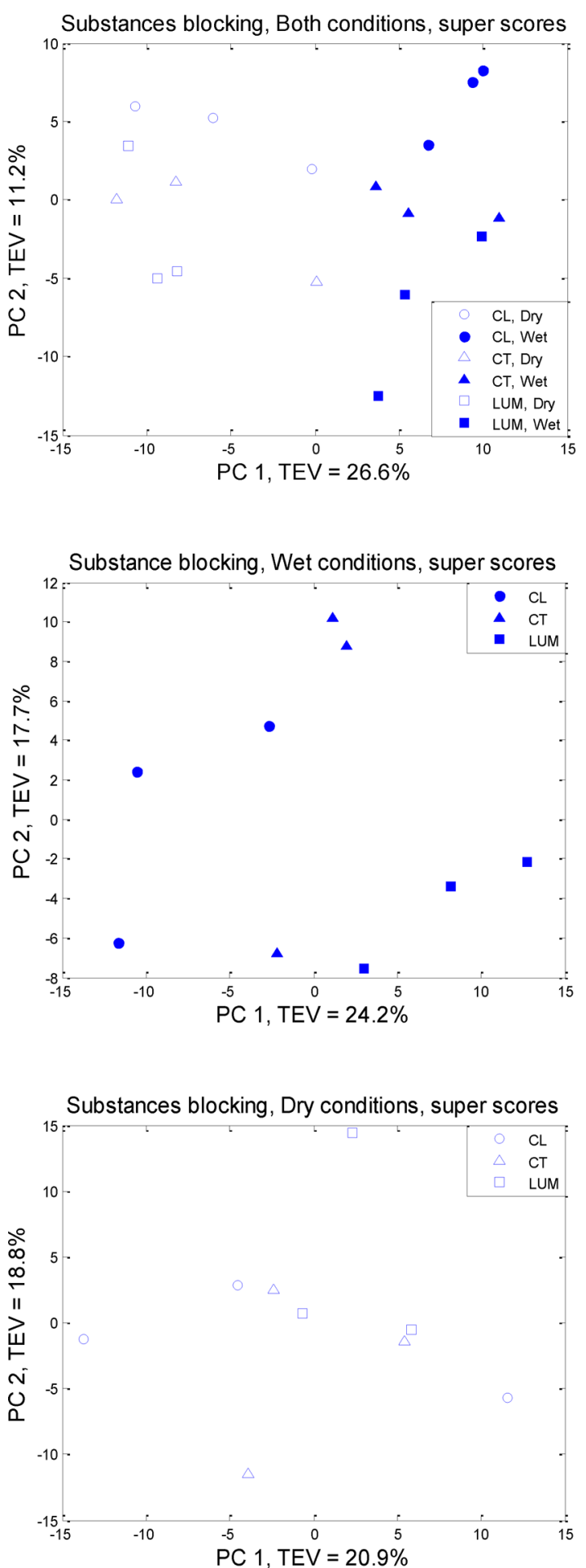

(c)

Figure 3. Super scores plots of the MB-PCA models based on substances blocking: (a) substances blocking with both conditions; (b) substances blocking with well-watered condition; (c) substances blocking with water-limited condition. TEV, total explained variance.

between the five different substances was very weak indeed, and a nonsignificant $p$ value of 0.470 was obtained from the corresponding permutation test. By contrast, the scores plots obtained from the location submatrix and the water treatment submatrix showed clear separations between the classes. The $p$ value of the scores of the location submatrix was 0.002 , and that of the water treatment matrix was also highly significant at $<0.001$ (i.e., not a single case of 1000 permutations had obtained higher


Figure 4. Scores plots of the three submatrices of the ASCA model.

SSQs than the observed one). The figures of the observed SSQs superimposed on the corresponding null distributions are given in Supplementary Figure S1 (Supporting Information). In summary, ASCA confirmed the results derived through MBPCA that water treatment was the factor with the most significant impact on grain composition, followed by location, whereas there was significant substance effect.

Conclusion. The compositional equivalence of transgenic drought-tolerant maize hybrids to a near-isogenic conventional 
comparator was demonstrated, and additional insights on the effect of drought on maize grain composition were offered. In this study, the effect of genetic modification was contrasted to other experimental factors: water treatment and location. Using the PCA variants MB-PCA and ASAC, it was demonstrated that water treatment had the greatest effect on composition followed by location; moreover, location effects were greater under well-watered conditions than under water-limited conditions. The multivariate options showed no significant differences between the drought-tolerant hybrids and the nearisogenic control, and both options corroborated results from the univariate analysis. This study represents the first application of MB-PCA and ASCA to crop compositional data. Results suggest these multivariate options are of value in unraveling coexisting multiple factors in a complex compositional study and thus present an opportunity to gain better views of each factor of interest whether these be germplasm, environment, management practices, or genetic modification.

\section{ASSOCIATED CONTENT}

\section{S Supporting Information}

Proximate, fiber, and mineral composition of forage (Supplementary Table 1) and individual site compositional data for the conventional control (Supplementary Tables 2-8) and Supplementary Figure $S 1$. This material is available free of charge via the Internet at http://pubs.acs.org.

\section{AUTHOR INFORMATION}

\section{Corresponding Authors}

*(R.G.) E-mail: roy.goodacre@manchester.ac.uk.

*(G.G.H.) E-mail: george.g.harrigan@monsanto.com.

\section{Funding}

R.G. is grateful to the UK BBSRC for financial support of metabolomics.

\section{Notes}

The authors declare no competing financial interest.

\section{ACKNOWLEDGMENTS}

We thank the Monsanto Field Agronomy group and the many field cooperators for conducting field trials, the Monsanto Product Characterization group for the molecular characterization of the test and control substances, and Monsanto's Sample Preparation Group for preparing maize samples for analysis.

\section{REFERENCES}

(1) Castiglioni, P.; Warner, D.; Bensen, R. J.; Anstrom, D. C.; Harrison, J.; Stoecker, M.; Abad, M.; Kumar, G.; Salvador, S.; D’Ordine, R.; Navarro, S.; Back, S.; Fernandes, M.; Targolli, J.; Dasgupta, S.; Bonin, C.; Luethy, M. H.; Heard, J. E. Bacterial RNA chaperones confer abiotic stress tolerance in plants and improved grain yield in maize under waterlimited conditions. Plant Physiol. 2008, 147, 446-455.

(2) Harrigan, G.; Ridley, W. P.; Miller, K. D.; Sorbet, R.; Riordan, S. G.; Nemeth, M. A.; Reeves, W.; Pester, T. A. The forage and grain of MON 87460 , a drought tolerant corn hybrid, are compositionally equivalent to that of conventional corn. J. Agric. Food Chem. 2009, 57, 9754-9763.

(3) Drury, S. M.; Reynolds, T. L.; Ridley, W. P.; Bogdanova, N.; Riordan, S.; Nemeth, M. A.; Sorbet, R.; Trujillo, W. A.; Breeze, M. L. Composition of forage and grain from second-generation insectprotected corn MON 89034 is equivalent to that of conventional corn (Zea mays L.). J. Agric. Food Chem. 2008, 56, 4623-4630.

(4) Ridley, W. P.; Sidhu, R. S.; Pyla, P. D.; Nemeth, M. A.; Breeze, M. L.; Astwood, J. D. Comparison of the nutritional profile of glyphosatetolerant corn event NK603 with that of conventional corn (Zea mays L.). J. Agric. Food Chem. 2002, 50, 7235-7243.
(5) McCann, M. C.; Trujillo, W. A.; Riordan, S. G.; Sorbet, R.; Bogdanova, N. N.; Sidhu, R. S. Comparison of the forage and grain composition from insect-protected and glyphosate-tolerant MON 88017 corn to conventional corn (Zea mays L.). J. Agric. Food Chem. 2007, 55, 4034-4042.

(6) Zhou, J.; Harrigan, G. G.; Berman, K. H.; Webb, E. G.; Klusmeyer, T. H.; Nemeth, M. A. Stability of the compositional equivalence of grain from insect-protected corn and seed from herbicide-tolerant soybean over multiple seasons, locations and breeding germplasms. J. Agric. Food Chem. 2011, 59, 8822-8828.

(7) Harrigan, G. G.; Lundry, D.; Drury, S.; Ridley, W. P.; Riordan, S. G.; Nemeth, M. A. Natural variation in crop composition and the impact of biotechnology. Nat. Biotechnol. 2011, 28, 402-404.

(8) Herman, R. A.; Price, W. D. Unintended compositional changes in genetically modified (GM) crops: 20 years of research. J. Agric. Food Chem. 2013, 61, 11695-11701.

(9) Harrigan, G. G.; Harrison, J. M. Assessing compositional variability through graphical analysis and Bayesian statistical approaches; case studies on transgenic crops. Biotechnol. Genet. Eng. Rev. 2012, 28, 15-32.

(10) Harrison, J. M.; Breeze, M. L.; Harrigan, G. G. Introduction to Bayesian statistical approaches to compositional analyses of transgenic crops 1. Model validation and setting the stage. Regul. Toxicol. Pharmacol. 2012, 60, 381-388.

(11) Harrison, J. M.; Breeze, M. L.; Berman, K. H.; Harrigan, G. G. Bayesian statistical approaches to compositional analyses of transgenic crops. 2. Application and validation of informative prior distributions. Regul. Toxicol. Pharmacol. 2013, 61, 251-258.

(12) Harrison, J. M.; Culp, D.; Harrigan, G. G. Bayesian MCMC analyses for regulatory assessments of food composition. Proceedings of Kansas State University Conference on Applied Statistics in Agriculture, Manhattan, KS, USA, 2013; pp 73-87.

(13) Harrison, J. M.; Howard, D.; Malven, M.; Halls, S. C.; Culler, A. H.; Harrigan, G. G.; Wolfinger, R. D. Principal variance component analysis of crop composition data: a case study on herbicide-tolerant cotton. J. Agric. Food Chem. 2013, 61, 6412-6422.

(14) Harrigan, G. G.; Culler, A. H.; Culler, M.; Breeze, M. L.; Berman, K. H.; Halls, S. C.; Harrison, J. M. Investigation of biochemical diversity in a soybean lineage representing 35 years of breeding. J. Agric. Food Chem. 2013, 61, 10807-10815.

(15) Xu, Y.; Goodacre, R. Multiblock principal component analysis: an efficient tool for analyzing metabolomics data which contain two influential factors. Metabolomics 2012, 8, 37-51.

(16) Zwanenburg, G.; Hoefsloot, H. C. J.; Westerhuis, J. A.; Jansen, J. J.; Smilde, A. K. ANOVA-principal component analysis and ANOVAsimultaneous component analysis: a comparison. J. Chemom. 2011, 25, 561-567.

(17) Feil, B.; Moser, S. B.; Jampatong, S.; Stamp, P. Mineral composition of the grains of tropical maize varieties as affected by pre-anthesis drought and rate of nitrogen fertilization. Crop Sci. 2005, $45,516-523$.

(18) Harder, G. J.; Carlson, R. E.; Shaw, R. H. Yield, yield components, and nutrient content of corn grain as influenced by post-silking moisture stress. Agron. J. 1982, 74, 275-278. 